\title{
Social Influence and mPOS Use: The Effects of Gender
}

\author{
Manuela Gutierrez-Leefmans ${ }^{1^{*}}$, Sunday Adewale Olaleye ${ }^{2}$
}

\begin{abstract}
Mobile points of sale become popular as countries migrate to cashless approaches. Small and medium enterprises embrace the technology, and users are attracted to it due to its benefits compared to traditional points of sale. However, scant literature refers to its use in emerging countries despite the relevance of small companies for such economies. This research presents a model of mobile point of sale use in Mexico based on a survey of graduate and undergraduate students. The study uses structural equation modeling to relate variables such as awareness and social influence with gender interaction. Results indicate that social influence influences awareness of the technology and is higher in males than in females. This study suggests that the mobile point of sale service providers in Mexico should consider social influence in their advertising. As users, men should be aware that social influence may influence their decision to use the technology.
\end{abstract}

Keywords: mobile point of sale; cashless; technology use; awareness; social influence; gender; emerging country; financial inclusion, small and medium enterprise

Submitted: August $4^{\text {th }}, 2021 /$ Approved: December $1^{\text {st }}, 2021$

\section{Introduction}

mPOS is an electronic device, which uses a bank card to process payments for goods and services through a mobile phone using an internet connection. This payment innovation is different from traditional points of sale, where credit or debit cards were used to process payments using a large device that produced a printed paper slip. On the one hand, mobile technology brings a simple and inexpensive way for small businesses to process payments. On the other hand, mPOS helps users carry less cash than some existing payment platforms. For a bank account owner, the type of point-of-sale terminal used is probably indifferent. The question is, what motivates users to embrace mPOS technology then? One reason may be the concern for the environment, as tickets for each transaction via mPOS are sent to the user's e-mail address. Such concern may be present in certain users with a "modern" attitude to card payments, such as young students.

There is literature on mPOS, especially in developed countries and some emerging ones. Mexico is a country with a vast population and many small and medium enterprises (SMEs), where mPOS technology has been around for about a decade. Frequent insecurity issues in the country related to organized crime and, more recently, the COVID-19 pandemic are making users choose to use less cash. Consumers are certainly using cards for payments more frequently and even for small transactions (Polasik et al. 2012), driven by more people with access to banking cards in emerging market economies and by greater availability of point-of-sale terminals. Lower-cost smartphone or tablet-based POS terminals have emerged, encouraging even smaller businesses to invest in them (Polasik et al., 2012). However, besides the economic incentive behind cashless technologies, social factors may influence users and contribute to their adoption.

Micro, small, and medium enterprises represent $99.8 \%$ of total business units in the country (INEGI, 2020). mPOS technology has the advantage of providing access to cheaper payment methods (the cost of an mPOS terminal can be as low as 200 Mexican pesos (10 USD) with commission starting at $3 \%$, quick processing times (cash available within three days) without having to deal with a bank. Some mPOS terminals allow the business owner to defer payments, which represents an advantage for the customer. If we also consider the vast young population in the country and the global high increase of mobile phone ownership, the study of mPOS becomes relevant.

Previous studies have been concerned with the study of new technologies to replace cash. Polasik et al. (2012) analyzed payment methods from cash and standard cards, contactless cards, RFID stickers, and mobile payments (NFC and remote). These authors found that the most popular electronic payment method in history was contactless cards (used offline and without printing paper slips). Also, information systems researchers have found that social influence is an essential factor that influences technology adoption. The socio-economic level is relevant in this respect, as $53 \%$ of adults in the country do not hold a bank card (Forbes, 2021), and there is still a traditional use of cash.

Understanding the factors that affect the use of mPOS technology among young Mexican consumers is helpful as the technology is relatively recent in the market. Hence, our research question: What is the impact of social influence and awareness on MPOS use in the context of a developing economy such as Mexico? Moreover, what role does gender play in such social influence? We developed a survey with 249 responses and used structural equation modeling (SEM) to confirm the critical role of social influence on mPOS use among young adults in Mexico. The first part of this paper presents a review of the literature on mPOS, focusing on the variables of awareness, social influence, and gender. The methodology section explains the sample, questionnaire

(1) Marketing Department of the Universidad de las Américas Puebla, México

(2) School of Business, JAMK University of Applied Sciences, Finland

*Corresponding author: maria.gutierrez@udlap.mx 
design, and measurement model. Finally, the paper presents the results and a discussion and conclusion section with practical implications and further research suggestions.

\section{Literature review}

\subsection{Use of Mobile Point of Sale (mPOS)}

Diverse research streams have used well-known theories to explain the adoption and use of mPOS technology, such as Unified Theory of Acceptance and Use of Technology Model (UTAUT) and Technology Acceptance Model (TAM). This study presents a model of mPOS use in an emergent country using awareness, social influence, and gender as a moderating variable. The three variables proposed are used to hypothesize on the use of mPOS technology due to their proven relevance in the mobile technology context.

\subsection{Awareness}

Rogers (1995) defined awareness as the user's knowledge about the capabilities of technology, its features, potential use, and cost and benefits. According to Mutahar et al. (2018, p. 184), "awareness implying knowledge about a technology and its benefits, and is, therefore, a key factor in the voluntary use of systems," and such voluntary use is related to its adoption. Also, according to Muthar et al. (2018), such awareness is likely to reduce the level of the risk perceived as well.

Studies on information systems awareness are numerous. Velmurugan and Velmurugan (2016) consider consumer's awareness of 3G mobile technology in India to study its adoption. This study finds that consumer awareness does indeed influence technology adoption together with ease of use. Mutahar et al. (2018) study mobile banking in Yemen and find an effect on perceived risk and awareness as external variables of the TAM. Also, a lack of awareness and understanding of the benefits of mobile banking services hinders acceptance and adoption (Laforet and $\mathrm{Li}, 2005)$. Following Ilesanmi (2012), where there is awareness, the penetration of a system will be high.

Research on technology awareness is vital to understand the behavioral intention to use technology. For example, Charbaji and Mikdashi (2003) empirically investigated the adoption factors that influence egovernment services among Lebanese postgraduate students, finding that awareness significantly influenced the intention to use e-government. Likewise, Rehman et al. (2012) empirically examined the adoption of e-government services among internet users in Pakistan, also finding that awareness significantly influences behavioral intention.

Awareness has also been found to influence women's membership in table banking groups, which increases the adoption of mobile payments services in Kenya (Gichuki and Mulu-Mutuku, 2018). The same study also established that women's control of business finances and decision-making significantly impact awareness and usage of mobile money technologies.

Specific research on POS conducted by Yaqub et al. (2013) posits that the reason for the slow adoption of e-payment in Nigeria is a lack of awareness of the system's advantages. These authors stressed a need for awareness to aid in the diffusion of POS in Nigeria (Ilesanmi, 2012). The higher the awareness, the higher the diffusion of the POS. By extension, the lower the awareness, the lower the diffusion (Abubakar and Ahmad, 2013). Abubakar and Ahmad's (2013) work confirms that technology awareness moderates the relationship between performance expectancy, effort expectancy, social influence, facilitating conditions, and the behavioral intention to use POS terminals.

The awareness of the benefits of mPOS is significant since it determines users' behavior. Therefore, awareness cannot be ignored when studying the adoption of technology such as mPOS in an emergent country. Hence, the following research hypothesis is proposed:

H1: Awareness has a positive impact on the use of mPOS.

Figure 1. Proposed framework for mPOS use

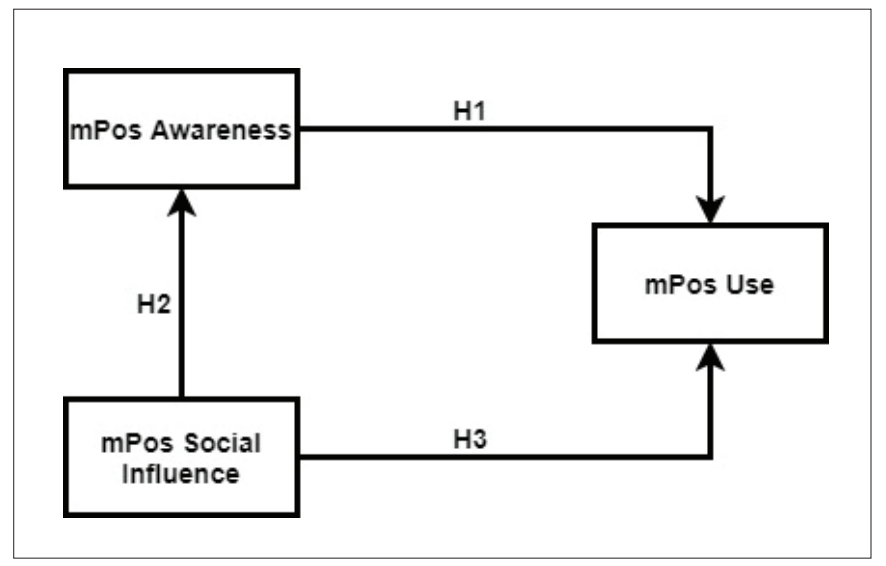

\subsection{Social influence}

Previous research found that original models such as TAM did not account for social influence (Malhotra and Galletta, 1999), viewing this as a limitation. Venkatesh et al. (2003) stated that performance expectancy, effort expectancy, social influence, and facilitating conditions were determinants of behavioral intention or use behavior. Social influence is defined as the extent to which a person perceives that important other believe he or she should use a new information system (Venkatesh et al., 2003). It can also be defined as the pressure exerted on the individual by the opinions of others (Van Biljon and Kotze, 2008). Interestingly, Van Biljon and Kotze (2008) divided social influence into human nature influences (inherited) and cultural (learned) influences.

Other researchers have found that social influence is significant in shaping personal intention to use new technology (Venkatesh and Davis, 2000). Yang and Forney (2013) adapt the UTAUT model to examine whether consumers' levels of technology anxiety moderate the causal relationships among determinants of mobile shopping adoption. They find that consumers with a high level of technology anxiety rely more on social influence in mobile shopping than consumers with a low level of it. Similarly, Da Silva et al. (2009) study explains the importance of social influence in mobile adoption in two modes: one related to pressure on individuals to adopt technology, and another related to benefits via social networks tied in with business networks. 
In a service context, technology research-based service supports social influence as a strong determinant of using the service (Taylor and Todd 1995). This service context has been widely studied in research that shows the relevance of online reviews. Researchers agree that the expectations and perceptions of significant others, such as friends, family, and colleagues, contribute to the social factors that affect an individual consumer's decision-making and perception towards a specific behavior (Phau and Teah 2009). In particular, consumer adoption of high technology is influenced by socialization forces associated with the desire to follow referent group norms (Kulviwat et al., 2009). We, therefore, propose the following research hypothesis:

$\mathrm{H} 2$ : Social influence will influence the behavioral intention to use mPOS.

\subsection{Gender}

The moderating effect of gender has been demonstrated in the adoption of e-commerce (Hwang, 2010). These studies show behavioral differences in online purchasing among users, according to their gender. Van Slyke et al. (2002) find that men have a greater tendency to make planned purchases (such as e-hardware), while women tend to buy other types of products or to make less planned purchases (like food and drinks). However, research on technology adoption that studies the moderating effect of gender has found contrasting results.

According to the findings of previous researchers (Venkatesh and Morris, 2000; Venkatesh et al., 2003), gender has proved to play a moderating role in the influence of performance expectancy on behavioral intention, and that the effect will be more substantial for men. It has also been suggested that women tend to be more sensitive to the influence of others, and therefore, social influence will be more salient when women form an intention to use new technology (Venkatesh et al., 2003). That is, finding that gender, age, experience, and voluntariness of use have moderating effects in accepting technology (Venkatesh et al., 2003).

Wang and Wang (2010) use the UTAUT model to understand whether or not there are gender differences in the acceptance of m-Internet. They find that although the main effects of perceived value and social influence on behavioral intention were significant, there were no gender differences. They also find that performance expectancy on behavioral intention was significant for men but non-significant for women. Also, effort expectancy was a more vital determinant of intention for women than for men, confirming the findings of previous studies (Venkatesh and Morris, 2000; Venkatesh et al., 2000, 2003).

Other studies are neutral regarding the gender variable. Li et al. (2008) study the impact of gender differences on the adoption and use of $\mathrm{m}$-commerce, finding that both genders had a similar adoption rate. They found that both groups adopted a similar staged pattern in their use of m-commerce services. According to their study, the only salient difference was that males move through the adoption stages at a more rapid rate than females do. Faqih and Jaradat (2015) confirm such neutrality finding that gender does not have any moderation effect on the adoption process of mobile commerce technology.
Regarding mobile payment, Liébana-Cabanillas et al. (2014) showed that the impact of the ease of use on the usefulness of the payment system was higher among men than among women, this way confirming previous studies, The authors suggested that the higher the ease of use of the tool, the greater the usefulness perceived by men. Liébana-Cabanillas et al. (2014) also confirm that the perceived usefulness by men will increase their intention to use, more than in the case of women. Therefore, the relevance of gender, given its moderating effect on the intention to use m-payment, was confirmed.

In a mobile banking context, gender has been linked to cultural issues. Goh and Sun (2014) find that male Muslims favor status and values orientations, so the perceived self-expressiveness significantly influenced their acceptance of mobile banking. On the other hand, female Muslims prefer social and utilitarian orientations. Thus, their acceptance of mobile banking was significantly influenced by perceived usefulness and social norms.

Gender is tied to diverse variables ranging from performance expectancy to social influence. We focus on social influence and propose the following research hypothesis:

H3: Social influence perception on the use of mPOS by males is higher than in females.

\section{Methodology}

This study employed a mixed methodology to access the participant's rich experience and use the qualitative response to complement the quantitative respondents' opinion. First, an in-depth interview with an expert in the banking sector was conducted. This person has worked both for private and public institutions for about 25 years in the payment industry and is currently a manager of one the leading mPOS companies in the country. The interview output led to the identification of relevant factors that may affect mPOS use. In addition to literature, it helped to configure the survey to consumers.

Second, this study utilized quantitative methodology for the objectivity and accuracy of the results based on the opinions of the Mexican segment that uses mPOS. Inferring from the earlier study that used students' samples (Mallat, 2007), a student's sample is found useful for a technologically inclined study like mPOS. The students, due to their curiosity, are described as digital natives with high levels of multitasking.

\subsection{Questionnaire design}

The questionnaire was designed and administered in Spanish (the official language of Mexico) to ensure that the respondents understood the survey better. Qualtrics online platform for surveys was utilized for the questionnaire administration. The study adapts factors from the existing literature and utilized a 5-point Likert scale where 1 stands for strongly disagree and 5 for strongly agree. The survey was launched in April 2020 and ran for about 30 days. The study uses the awareness variable together with social influence and mPOS use. Likewise, it emphasizes the moderating effect of gender on social influence. 


\subsection{Sampling}

The study is based on a survey directed to undergraduate and graduate students from the Universidad de las Américas, Puebla (UDLAP), one of the country's best private universities in Mexico. The university has a student population of 10,000; the study has a 320-person sample size, with an $85 \%$ confidence level and a $4 \%$ margin of error. However, out of the 320-questionnaire administered, only 249 were relevant after the data cleaning process. The study used SPSS v. 26 for descriptive analysis, and the results show that the female participants are more than the male (female $=57 \%$, male $=43 \%$ ). Education-wise, the bachelor students recorded $83 \%$ while the masters account for $17 \%$. The participants aware of mPOS were $59 \%, 29 \%$ were not aware of it, while only $12 \%$ were not sure about the technology. $74 \%$ of the participants are interested in mPOS, and 26\% are not interested. $72 \%$ see $\mathrm{mPOS}$ as a path to a cashless economy and believed it has benefits, while $28 \%$ did not see any benefits in a cashless system. $43 \%$ of the participants have Near Field Communication enabled phones, while $57 \%$ do not have NFC-enabled phones. In the future, $74 \%$ of the participants will be willing to use NFC-enabled mPOS devices, while $26 \%$ are not willing (details in Table 1 ).

This study utilized SmartPLS ver. 3.3.3 software to analyze mPOS data, focused on structural equation modeling partial least square and tested the hypotheses with SmartPLS bootstrapping. The study also conducted moderation analysis with SmartPLS.

Table 1. Demographics and Psychographics

\begin{tabular}{lccc}
\hline Demographics and Psychographics & Criterion & Frequency & Percent \\
\hline Gender & Male & 107 & 43 \\
& Female & 142 & 57 \\
Education & Bachelor & 206 & 83 \\
& Masters & 43 & 17 \\
mPOS Awareness & Yes & 147 & 59 \\
& No & 72 & 29 \\
mPOS Interest & Not Sure & 30 & 12 \\
& Yes & 185 & 74 \\
Cashless System Benefits & No & 64 & 26 \\
& Yes & 179 & 72 \\
NFC enabled phone & No & 70 & 28 \\
Use of NFC enabled mPOS device & Yes & 106 & 43 \\
& No & 64 & 26 \\
\hline
\end{tabular}

\section{Results}

This study employed SmartPLS to predict mPOS use as the driver of user's awareness and social influence (Hair, Ringle, and Sarstedt, 2011). This section captures the results of the measurement and structural model. In the first step, the study examined the quality criteria of the mPOS data with SmartPLS, checked the structural relationship in the second step, and tested the formulated hypotheses. The measurement model shows that items under the awareness construct loaded well and above the threshold of 0.5 . Items 3 and 4 loaded below the threshold and were removed to have an accurate model (Hair, Anderson, Babin and Black, 2010).

Table 2. Factors Loading

\begin{tabular}{lccr}
\hline Items & Awareness & Social Influence & mPos Use \\
\hline Awareness1 & 0.854 & & \\
Awareness2 & 0.956 & & \\
SInfluence1 & & & \\
SInfluence3 & 0.928 & \\
mPOSUse1 & 0.941 & 0.932 \\
mPOSUse2 & & 0.970 \\
mPOSUse3 & & & 0.956 \\
\hline
\end{tabular}

Note. Awareness 3 and 4, Social Influence 2 and 4, mPOS Use

4 did not meet the thresholds of the factor loading.

Similarly, items 1 and 3 loaded well, and items 2 and 4 were removed while three items under mPOS use loaded and met the set thresholds. Only one item (4) did not meet the thresholds of the factor loading (details in Table 2). For construct reliability and validity, both Cronbach, rho_A, Composite reliability, and Average Variance Extracted (AVE) were examined in Table 3. The results show that the instrument adopted is reliable as the Cronbach loaded between $(0.798$ and 0.949$)$. The Cronbach values are above the threshold of 0.7 as proposed by Tavakol and Dennick, (2011). Both rho_A and Composite Reliability supported the Cronbach's results reliability. Also, the Average Variance Extracted meets the thresholds of 0.5, and the bolded diagonal values as the square root of AVE in Table 3 confirmed the convergent validity of the study while Tables 4 and 5 established discriminant validities of the study.

Table 3. Construct Reliability and Validity

\begin{tabular}{lrrrr}
\hline & $\begin{array}{r}\text { Cronbach's } \\
\text { Alpha }\end{array}$ & rho_A & $\begin{array}{r}\text { Composite } \\
\text { Reliability }\end{array}$ & $\begin{array}{r}\text { Average Variance } \\
\text { Extracted (AVE) }\end{array}$ \\
\hline Awareness & 0.798 & 0.986 & 0.902 & 0.822 \\
Social Influence & 0.856 & 0.861 & 0.933 & 0.874 \\
mPos Use & 0.949 & 0.951 & 0.967 & 0.908 \\
\hline
\end{tabular}

Table 4. Discriminant Validity

Fornell-Larcker Criterion

\begin{tabular}{lrcr}
\hline & Awareness & Social Influence & mPos Use \\
Awareness & $\mathbf{0 . 9 0 6}$ & & \\
Social Influence & 0.339 & $\mathbf{0 . 9 3 5}$ & \\
mPosUse & 0.480 & 0.443 & $\mathbf{0 . 9 5 3}$ \\
\hline
\end{tabular}

Table 5. Heterotrait-Monotrait Ratio (HTMT)

\begin{tabular}{lrrr} 
& Awareness & Social Influence & mPosUse \\
Awareness & $\mathbf{1}$ & & \\
Social Influence & 0.394 & $\mathbf{1}$ & \\
mPosUse & 0.514 & 0.486 & $\mathbf{1}$ \\
\hline
\end{tabular}


Table 6 shows the structural model of two independent variables (Awareness and Social Influence) and one dependent variable (mPOS Use). The current study shows that Hypotheses $1-3$ were above the thresholds of 1.96. H1 Awareness -> mPos Use ( $\beta=0.373$, $\mathrm{P}<0.001, f=0.18), \mathrm{H} 2$ Social Influence $\rightarrow$ Awareness $(\beta=0.339$, $\mathrm{P}<0.001, f=0.13), \mathrm{H} 3$ Social Influence $->\mathrm{mPos}$ Use $(\beta=0.316$, $\mathrm{P}=0.005, f=0.13$ )
$\mathrm{H} 4$ in Figure 3 shows the interaction effect of Social Influence ${ }^{\star}$ Gender $^{\star}$ mPos Use $(\beta=-0.321, \mathrm{P}<0.001, f=0.10)$. Awareness is the highest predictor of $\mathrm{mPOS}$ in this context. Among the variables, awareness has the largest effect size, while social influence as predictors of awareness and mPOS use had the same effect size. which indicates a small effect size. The mPOS Awareness explained $\left(\mathrm{R}^{2}\right) 11.2 \%$ while mPoS Use explained $\left(\mathrm{R}^{2}\right) 31.4 \%$ (Figure 2). Further, the study summarized the interview conducted with the mPOS company manager in Mexico and presents the excerpt in the discussion section.

Table 6. Path Coefficients (Mean, STDEV, T-Values, P-Values)

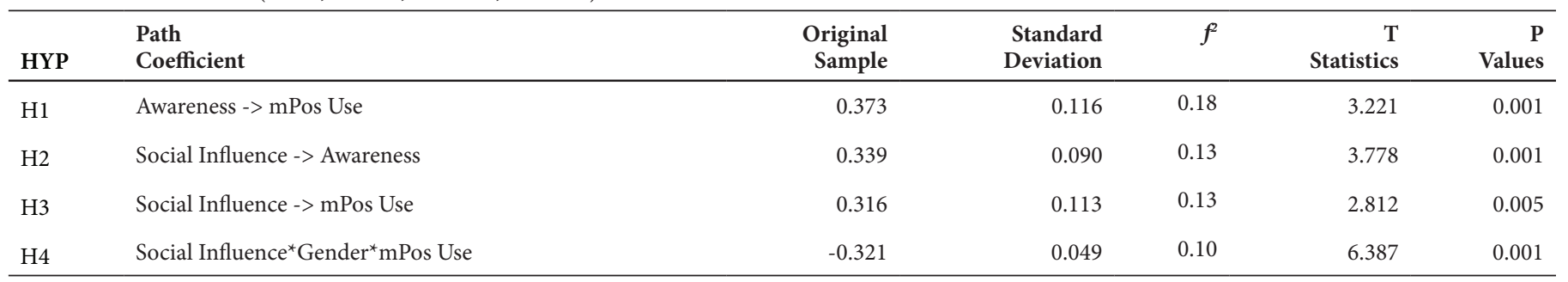

Figure 2. Results of the framework of mPOS

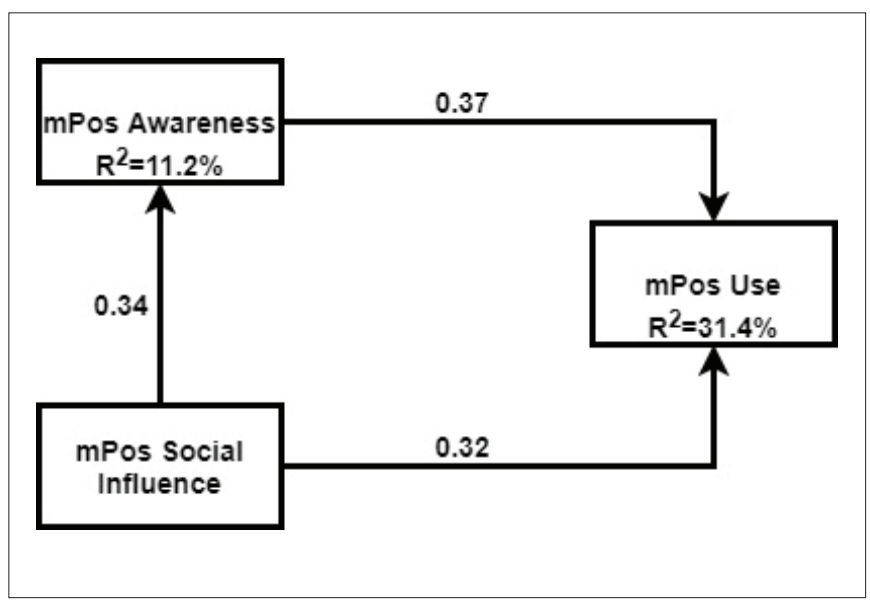

Figure 3. Interaction Effect of Social influence and mPOS use through Gender

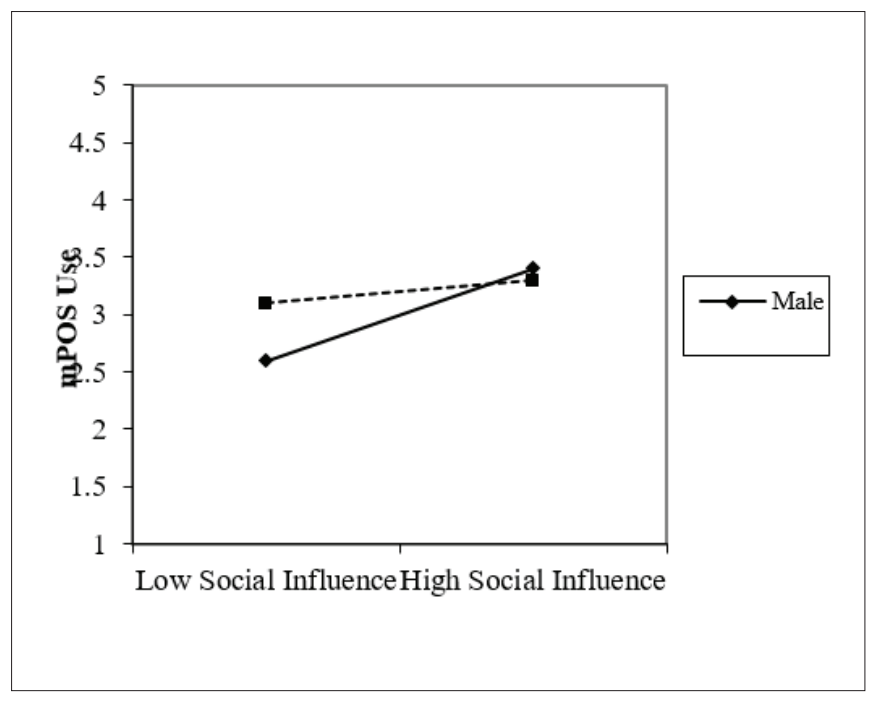

\section{Discussion and conclusions}

According to studies conducted by the interviewed mPOS company manager, millennials use little cash and do many daily transactions, making them an exciting segment to address. The manager reported a growth of about $80 \%$ in the use of mPOS in the country. This finding reflects the initiative of companies that sell the mPOS readers, who are concerned about financial inclusion in the country and see Mexico as a well-positioned country in terms of regulations. The large informal sector in the country, besides the high growth in mobile phone use throughout the country, make the business of mPOS attractive. On one side, merchants may prefer to receive cash due to tax regulations in the country. On the other side, mPOS companies deal with an intermediary bank leaving the small company free to manage their business. They provide reports, which help the SME owner with its accounting. Another advantage is that mobile terminals do not require a recurrent number of sales by banks, as traditional terminals do.

Therefore, it seems clear why merchants would adopt mPOS. On the other side are consumers who decide between using cash or conducting transactions with the mobile terminal. $\mathrm{mPOS}$ is an easy-to-use technology; however, probably not well known by users in Mexico, and there may be other factors driving consumers to use it. As reported by the manager, its company uses mainly social media to advertise as it is addressing young adults. This raised the question whether users are really aware of the technology and if social influence could be exerting an influence on $\mathrm{mPOS}$ awareness.

Small businesses and individuals move towards an alternative payment system in Mexico due to the existing banks' pricey fees and the bank's excessively complicated administrative procedure. However, despite the benefits of an alternative payment system, there is still a gap in mPOS adoption, use, and continuous use in Mexico. In their study, Din et al. (2013) employed descriptive, measurement, and structural statistics to explain the phenomenon of mPOS and to 
answer the research questions. The present study intends to know why social influence and gender compatibility are determinants of mPOS use in Mexico among young adults.

This study empirically shows that awareness and social influence have a significant impact on mPOS use while social influence impacts mPOS awareness and gives a theoretical understanding of factors responsible for mPOS use in the context of Mexico. This result is in line with the earlier study that established the importance of the awareness variable and mentioned that it is a crucial variable to study the attitude and behavior of the people (Din, Haron and Ahmad, 2013). It is essential to create a knowledge of mPOS in Mexico, especially for the illiterate merchants and other target group of users. There is an indication that people are willing to use mPOS in Mexico. This group of innovators can greatly influence early adopters, early majority, and even laggards. There is power in the life of influence, and the innovators can share their experience of mPOS to influence the undecided users. The mPOS managers can take advantage of the social influence of opinion leaders in Mexico to acquire more users through conformity, compliance, obedience, or minority influence.

This study also reveals the predominant role of gender and social influence as a change agent of mPOS use. The study established a strong moderation effect of gender and theoretically contributed that males have a stronger social influence than females in mPOS use. This study confirmed Mexico as a masculine culture. The male has upper social influence over their female counterpart. mPOS managers should put gender differences into consideration when planning their advertising campaigns and their segmentation strategy. Contrary to other research, social influence, particularly in males, resulted in an important influencer to the use of mPOS. Theoretically, this study integrates awareness, social influence, and the moderating effect of gender as drivers of mPOS use. Overall, the study contributed to advance existing literature on mobile payment.

\subsection{Practical implications and further research}

As expressed by the manager interviewed, mPOS technology is a way to address the bottom of the pyramid, those small businesses that otherwise would struggle to comply all requisites to have a direct relationship with a bank. To increase financial inclusion in the country, studies of this nature are important as they provide a guidance for mPOS developers, advertisers and SMEs owners.

Managerial-wise, this study suggests that the mPOS service providers in Mexico should pay closer attention to mPOS users' social influence. Besides, mPOS service providers should consider the social influence and gender when addressing their product to a specific customer segment. Male users should also be aware that the decision to use mPOS may be related to specific social influence.

This study was limited to university students and a single country since mPOS disruption is a global phenomenon. Further research should test the model of this study in other countries and other age groups. The researcher should add psychological variables such as anxiety, mood, and satisfaction to add an emotional attachment to
mPOS linked to gender. Future researchers should link this study with the merchants being the focal users of mPOS and examine the merchant' use and continuous use of mPOS.

\section{Aknowledgment}

The authors would like to thank the mPOS company manager interviewed for his helpful insights to develop this study.

\section{References}

Abubakar, F. M., \& Ahmad, H. B. (2013). The moderating effect of technology awareness on the relationship between UTAUT constructs and behavioural intention to use technology: A conceptual paper. Australian Journal of Business and Management Research, 3(2), 14-23.

Charbaji, A., \& Mikdashi, T. (2003). A path analytic study of the attitude toward e-government in Lebanon. Corporate Governance, 3(1), 76-82.

Din, N., Haron, S., \& Ahmad, H. (2013). The level of awareness on the green ICT concept and self directed learning among Malaysian Facebook users. Procedia-Social and Behavioral Sciences, 85, 464-473.

Faqih, K. M., \& Jaradat, M. I. R. M. (2015). Assessing the moderating effect of gender differences and individualism-collectivism at individual-level on the adoption of mobile commerce technology: TAM3 perspective. Journal of Retailing and Consumer Services, 22, 37-52.

Gichuki, C. N., \& Mulu-Mutuku, M. (2018, March). Determinants of awareness and adoption of mobile money technologies: Evidence from women micro entrepreneurs in Kenya. In Women's Studies International Forum (67), 18-22. Pergamon.

Goh, T. T., \& Sun, S. (2014). Exploring gender differences in Islamic mobile banking acceptance. Electronic Commerce Research, 14(4), 435-458.

Hair, J. F., Anderson, R. E., Babin, B. J., \& Black, W. C. (2010). Multivariate data analysis: A global perspective (Vol. 7).

Hair, J. F., Ringle, C. M., and Sarstedt, M. 2011. "PLS-SEM: Indeed a Silver Bullet." Journal of Marketing Theory and Practice, 19 (2): 139-151.

Hwang, Y. (2010), “The moderating effects of gender on e-commerce systems adoption factors: an empirical investigation", Computers in Human Behavior, 26, 1753-1760

Ilesanmi, F. (2012, September 10). Cashless Policy: Disconnects Must be Fixed - Adeyemi | Nigeria CommunicationsWeek. Retrieved from Communicationsweek Media Limited (Nigeria). Retrieved from www.nigeriacommunicationsweek.com.ng/e-guest/cashless-policydisconnects-must-be-fixed-adeyemi

INEGI (2020, June 25) Estadísticas a propósito del día de las Micros, Pequeñas y Medianas Empresas Comunicado de Prensa. From https:// www.inegi.org.mx/contenidos/saladeprensa/aproposito/2020/MYPIMES20.pdf 
Kulviwat, S., G. C. Bruner II, and O. Al-Shuridah (2009) "The Role of Social Influence on Adoption of High Tech Innovations: The Moderating Effect of Public/Private Consumption," Journal of Business Research, 62 (7), 706-712

Laforet, S. and Li, X. (2005) 'Consumers' attitudes towards online and mobile banking in China', International Journal of Bank Marketing, 23 (5), 362-380, https://doi.org/ 10.1108/02652320510629250

Liébana-Cabanillas, F. J., Sánchez-Fernández, J., \& Muñoz-Leiva, F. (2014). Role of gender on acceptance of mobile payment. Industrial Management \& Data Systems.114 (2), 220-240. https://doi. org/10.1108/IMDS-03-2013-0137

Mallat, N. (2007). Exploring consumer adoption of mobile payment - A qualitative study. Journal of Strategic Information Systems, 16(4), 413-432.

Mutahar, A. M., Daud, N. M., Thurasamy, R., Isaac, O., \& Abdulsalam, R. (2018). The Mediating of Perceived Usefulness and Perceived Ease of Use: The Case of Mobile Banking in Yemen. International Journal of Technology Diffusion (IJTD), 9(2), 21-40.

Phau, I. and M. Teah (2009) "Devil Wears (counterfeit) Prada: A Study of Antecedents and Outcomes of Attitudes towards Counterfeits of Luxury Brands," Journal of Consumer Marketing, 26 (1) 15-27

Polasik, M., Górka, J., Wilczewski, G., Kunkowski, J., Przenajkowska, K., \& Tetkowska, N. Time efficiency of Point-of-Sale payment methods: Empirical results for cash, cards and mobile payments. In International Conference on Enterprise Information Systems (pp. 306320). Springer, Berlin, Heidelberg (2012).

Rehman, M., Esichaikul, V., \& Kamal, M. (2012). Factors influencing e-government adoption in Pakistan. Transforming Government: People, Process and Policy, 6(3), 258-282.

Rogers, E. M. (1995). Diffusion of innovations. New York, NY: Free Press, Simon and Schuster
Sandoval-Meza (2021) México en el top 5 de países menos bancarizados. Retreived from https://www.forbes.com.mx/mexico-en-el-top5-de-paises-menos-bancarizados/

Tavakol, M., \& Dennick, R. (2011). Making sense of Cronbach's alpha. International journal of medical education, 2, 53.

Taylor, S. and P. Todd, "Understanding Information Technology Usage: A Test of Competing Models," Information Systems Research, 6 (4),144-176, 1995b

Van Slyke, C., Comunale, C.L. and Belanger, F. (2002), "Gender differences in perceptions of web-based shopping", Communications of the ACM, 45 (7), 82-86.

Velmurugan, M. S., \& Velmurugan, M. S. (2016). Information technology adoption on $3 \mathrm{G}$ mobile phones in India: the empirical analyses with SPSS 20, SmartPLS2. 0M3 and LISREL8. 80-Part 1. International Journal of Business Innovation and Research, 11(4), 484-511.

Venkatesh, V., \& Morris, M. G. (2000). Why don't men ever stop to ask for directions? Gender, social influence, and their role in technology acceptance and usage behavior. MIS Quarterly, 115-139.

Venkatesh, V., Morris, M. G., Davis, G. B., \& Davis, F. D. (2003). User acceptance of information technology: Toward a unified view. MIS Quarterly, 27(3), 425-478

Wang, H. Y., \& Wang, S. H. (2010). User acceptance of mobile internet based on the unified theory of acceptance and use of technology: Investigating the determinants and gender differences. Social Behavior and Personality: an Internationaljjournal, 38(3), 415-426

Yang, K., \& Forney, J. C. (2013). The moderating role of consumer technology anxiety in mobile shopping adoption: differential effects of facilitating conditions and social influences. Journal of Electronic Commerce Research, 14(4), 334.

Yaqub, J., Bello, H. T., Adenuga, I. A., \& Ogundeji, M. O. (2013). The Cashless Policy in Nigeria: Prospects and Challenges. International Journal of Humanities and Social Science, 3(3), 200-212 
